\title{
FSR 584 - a new globular cluster in the Galaxy?
}

\author{
E. Bica ${ }^{1}$, C. Bonatto ${ }^{1}$, S. Ortolani ${ }^{2}$, and B. Barbuy ${ }^{3}$ \\ 1 Universidade Federal do Rio Grande do Sul, Departamento de Astronomia, CP 15051, RS, Porto Alegre 91501-970, Brazil \\ e-mail: [charles;bica]@if.ufrgs.br \\ 2 Università di Padova, Dipartimento di Astronomia, Vicolo dell'Osservatorio 5, 35122 Padova, Italy \\ e-mail: sergio.ortolani@unipd.it \\ 3 Universidade de São Paulo, Departamento de Astronomia, Rua do Matão 1226 São Paulo 05508-900, Brazil \\ e-mail: barbuy@astro.iag.usp.br
}

Received 6 June 2007 / Accepted 22 June 2007

\begin{abstract}
Aims. We investigate the nature of the recently catalogued star cluster candidate FSR 584, which is projected in the direction of the molecular cloud W 3 and may be the nearest globular cluster to the Sun.

Methods. 2MASS colour-magnitude diagrams, the stellar radial density profile, and proper motions are employed to derive fundamental and structural parameters.

Results. The colour-magnitude diagram morphology and the radial density profile show that FSR 584 is an old star cluster. With proper motions, the properties of FSR 584 are consistent with a metal-poor $([\mathrm{Fe} / \mathrm{H}] \approx-2)$ globular cluster with a well-defined turnoff and evidence of a blue horizontal-branch. FSR 584 might be a Palomar-like halo globular cluster that is moving towards the Galactic plane, currently at $\approx 20 \mathrm{pc}$ above it. The distance from the Sun is $d_{\odot} \approx 1.4 \mathrm{kpc}$, and it is located at $\approx 1 \mathrm{kpc}$ outside the Solar circle. The radial density profile is characterized by a core radius of $R_{\text {core }}=0.3 \pm 0.1 \mathrm{pc}$. However, we cannot exclude the possibility of an old open cluster.

Conclusions. Near-infrared photometry coupled to proper motions support the scenario where FSR 584 is a new globular cluster in the Galaxy. The absorption is $A_{\mathrm{V}}=9.2 \pm 0.6$, which makes it a limiting object in the optical and explains why it has so far been overlooked.
\end{abstract}

Key words. Galaxy: globular clusters: individual: FSR 584

\section{Introduction}

Since they are long-lived, globular clusters (GCs) formed in the initial phases of the Galaxy may preserve information in their structure and spatial distribution that is essential for probing the early Milky Way physical conditions. Derivation of the present-day spatial distribution of GCs, as well as their physical and chemical properties, is important to better understand the formation and evolution processes, and trace the geometry of the Galaxy (Mackey \& van den Bergh 2005; Bica et al. 2006b).

Over a Hubble time, the structure of GCs, especially the less-massive ones, is affected by external processes such as the Galactic tidal stress and dynamical friction, and internal ones such as mass loss by stellar evolution, mass segregation and evaporation (e.g. Khalisi et al. 2007; Lamers et al. 2005; Gnedin $\&$ Ostriker 1997). The net result of these processes is a decrease in the mass of the clusters that may accelerate the core collapse phase for some (Djorgovski \& Meylan 1994, and references therein). The bottom line is that internal dynamical processes are affected by external ones, whose strength also depends on the GC Galactocentric distance (e.g. Djorgovski \& Meylan 1994; van den Bergh 1991; Chernoff \& Djorgovski 1989).

As remarked by van den Bergh (1991), the Galactic halo is a friendly environment to clusters of any size. Small GCs formed in the halo should have a high probability of surviving to date, and the scarcity of both small halo GCs and large ones close to the Galactic center could result from a physical correlation established at cluster formation. Thus, the discovery and characterization of low-mass GCs plays an important role, both to improve the statistics at the faint-end of the GC-parameter distribution, and to set observational constraints on the clusterdynamical processes discussed above.

The number of known GCs in the Galaxy has been slowly increasing as deeper surveys have been carried out. The compilation of Harris (1996, and the 2003 update $^{1}$ ) contains 150 GCs. Kobulnicky et al. (2005) discovered the far-IR GC GLIMPSEC01. Carraro (2005) identified Whiting 1 as a young halo GC. Two stellar systems detected with the Sloan Digital Sky Survey (SDSS) in the outer halo, Willman 1 (SDSS J1049+5103) and SDSS J1257+3419, might be GCs or dwarf galaxies (Willman et al. 2005; Sakamoto \& Hasegawa 2006). Ortolani et al. (2006) identified AL 3 as a new GC in the bulge with a blue horizontal branch (HB). More recently, Froebrich et al. (2007b) found evidence that FSR 1735 is a GC in the inner Galaxy, and Belokurov et al. (2007) found the faint halo GC SEGUE 1 using SDSS. Finally, Koposov et al. (2007) reported the discovery of two very-low luminosity halo GCs (Koposov 1 and 2) detected with SDSS.

In a recent observational effort to uncover potential star clusters, Froebrich et al. (2007a) carried out an automated search for stellar overdensities using the $2 \mathrm{MASS}^{2}$ database for $b<20^{\circ}$, which resulted in a list of 1021 candidates. Based on diagnostic diagrams involving number of stars, core radius and central density, 9 of these were classified as GC candidates, whilst the majority were open cluster candidates. FSR 584 is among the

\footnotetext{
1 http://physun.physics.mcmaster.ca/Globular.html

2 http://www.ipac. caltech.edu/2mass/releases/allsky/
} 


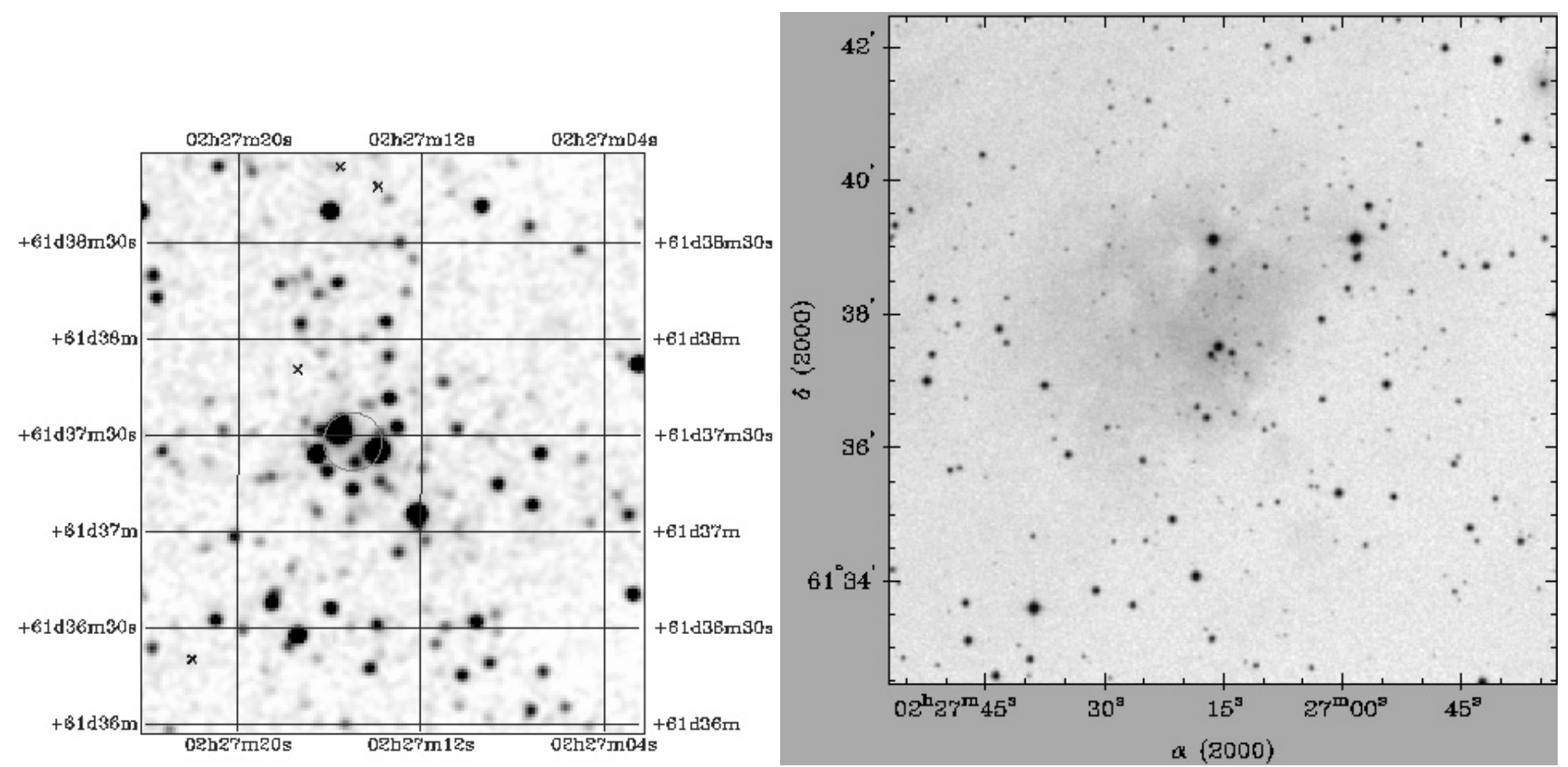

Fig. 1. Left: $3^{\prime} \times 3^{\prime} 2$ MASS $H$ image of FSR 584. The small circle marks the central region of the cluster (Sect. 2). North is up and East is left. Right: $10^{\prime} \times 10^{\prime}$ XDSS $R$ image, where an optical nebula of diameter of $\sim 2.5^{\prime} \approx 1$ pc (Sect. 3 ) is projected in the central cluster area.

latter. A systematic inspection of the respective 2MASS images revealed that the candidate FSR 584 is a relatively populous star cluster with a pronounced core. In fact, in the 2MASS atlas, the cluster image (Fig. 1) resembles a Palomar GC (e.g. Ortolani et al. 1985).

In the present work, we investigate the nature of FSR 584. In Sect. 2 we analyze near-IR colour-magnitude diagrams (CMDs), proper motions (PMs) and cluster structure. In Sect. 3 we discuss cluster properties. Concluding remarks are given in Sect. 4.

\section{Photometric parameters}

The original coordinates of FSR 584 (Froebrich et al. 2007a) are slightly shifted with respect to the central concentration of stars as seen in 2MASS images (Fig. 1). The revised values are $\alpha(J 2000)=02^{\mathrm{h}} 27^{\mathrm{m}} 15^{\mathrm{s}}$ and $\delta(J 2000)=61^{\circ} 37^{\prime} 28^{\prime \prime}$, which correspond to the Galactic coordinates $\ell=134.06^{\circ}$ and $b=+0.84^{\circ}$. We are dealing with a 2 nd quadrant cluster projected very close to the plane in Cassiopeia. The following analysis is based on 2MASS photometry and tools as described in Bonatto \& Bica (2007).

$J \times(J-H)$ CMDs of different extractions around the cluster center are presented in Fig. 2. Panel (a) shows the region $R<3^{\prime}$ that contains most of the cluster stars (Sect. 2.3). A disk-like population appears to merge into a redder component at $(J-H)>1$.1. Panel (b) contains an equal area annular extraction at $R \approx 20^{\prime}$. The disk component stands out as in (a) but the redder one is vanishing. The red component is essentially absent at $R \sim 30^{\prime}$ (panel $\mathrm{c}$ ).

\subsection{Field-star decontamination}

To disentangle the intrinsic CMD morphology from the field, we apply the decontamination algorithm described in Bonatto \& Bica (2007). The algorithm works on a statistical basis by measuring the relative number-densities of candidate field and cluster stars in small cubic CMD cells whose axes correspond to the magnitude $J$ and the colours $(J-H)$ and $\left(J-K_{\mathrm{s}}\right)$ (considering as well the $1 \sigma$ uncertainties in the 2MASS bands). These are the 2MASS colours that provide the maximum variance among CMD sequences for star clusters of different ages (e.g. Bonatto et al. 2004).

Basically, the algorithm (i) divides the full range of magnitude and colours of a given CMD into a 3D grid, (ii) computes the expected number-density of field stars in each cell based on the number of comparison field stars with magnitude and colours compatible with those in the cell, and (iii) subtracts the expected number of field stars from each cell. The algorithm results are sensitive to local variations of field-star contamination with colour and magnitude (Bonatto \& Bica 2007). Cell dimensions are $\Delta J=0.5$, and $\Delta(J-H)=\Delta\left(J-K_{\mathrm{s}}\right)=0.25$, which are large enough to allow sufficient star-count statistics in individual cells and small enough to preserve the morphology of the CMD evolutionary sequences. As a comparison field, we use the region $20<R\left(^{\prime}\right)<30$ around the cluster center to obtain representative background statistics.

Three different grid specifications in each dimension are used to minimize potential artifacts introduced by the choice of parameters. For instance, for a CMD grid starting at magnitude $J_{0}$ (and cell width $\Delta J$ ), additional runs for $J_{0} \pm \frac{1}{3} \Delta J$ are included. Considering the 2 colours as well, 27 different outputs are obtained, from which the average number of probable cluster stars $\left\langle N_{\mathrm{cl}}\right\rangle$ results. Typical standard deviations of $\left\langle N_{\mathrm{cl}}\right\rangle$ are at the $\approx 2.5 \%$ level. The final field star-decontaminated CMD contains the $\left\langle N_{\mathrm{cl}}\right\rangle$ stars with the highest number-frequencies. Stars that remain in the CMD after the decontamination are in cells where the stellar density presents a clear excess over the field. Consequently, they have a significant probability of being cluster members. Further details on the algorithm, including discussions on subtraction efficiency and limitations, are given in Bonatto \& Bica (2007).

The resulting field star decontaminated CMD derived from the extraction $R<3^{\prime}$ (panel a) is shown in panel (d). As 


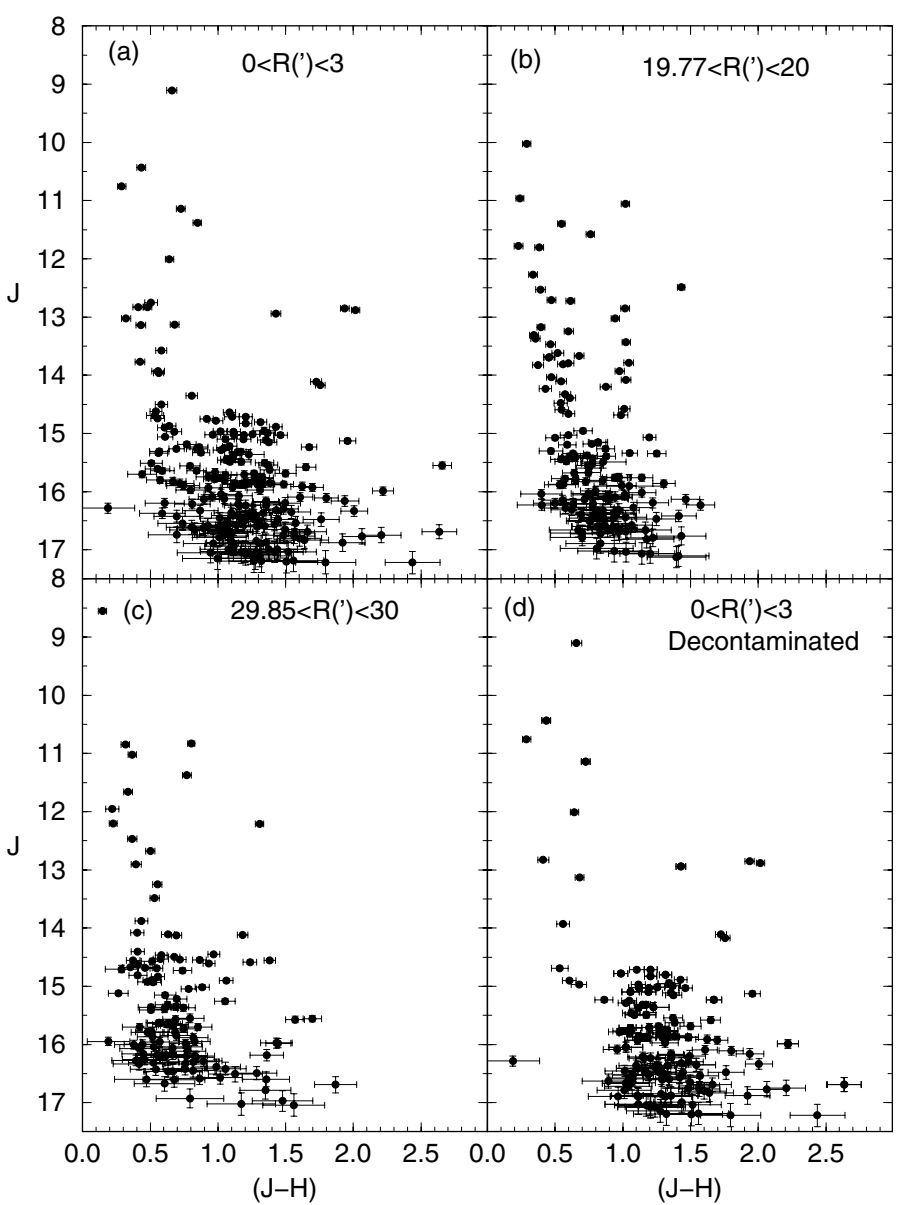

Fig. 2. Equal area CMDs extracted as a function of distance from the cluster center, as indicated in the panels. Photometric errors are shown. From a) to c) the disk component remains, while the red star content vanishes. Panel d): field decontaminated CMD of panel a).

expected, most of the disk component is removed, while a populous red component shows up. This extraction, which corresponds to the bulk of the cluster stars (see Fig. 5), indicates that we are dealing with a relatively populous star cluster, as suggested by the prominent main sequence (MS) at $1.0 \lesssim(J-H) \lesssim$ 2.0. Comparable results are derived with the additional 2MASS filter $K_{\mathrm{s}}$, by means of $J \times\left(J-K_{\mathrm{s}}\right)$ CMDs (Fig. 3).

\subsection{The central CMD and proper motions}

To minimize field contamination issues, we examine in Fig. 3 the observed CMD of a central $\left(R<1^{\prime}\right)$ extraction. A similar procedure was fundamental in the analysis that unveiled AL 3 as a GC (Ortolani et al. 2006). The central stellar density is so high with respect to the background that when applied to this CMD, the field decontamination would remove only 2 stars (Fig. 3). Besides the well-defined MS and turnoff (TO) sequences indicating old populations, the CMD displays a group of blue stars suggesting a blue HB. To test the possibility that FSR 584 is an old open cluster (OC), we compared its CMD morphology with that of the $\approx 7$ Gyr OC NGC 188 (Bonatto et al. 2005). The locus occupied by the probable HB stars in FSR 584 is very different from that of the blue-stragglers in NGC 188. Although hot HB candidates have been detected in old open clusters (Landsman et al. 1998), they are rare, whereas blue HB stars are typical of metal poor GCs. On the other hand, the

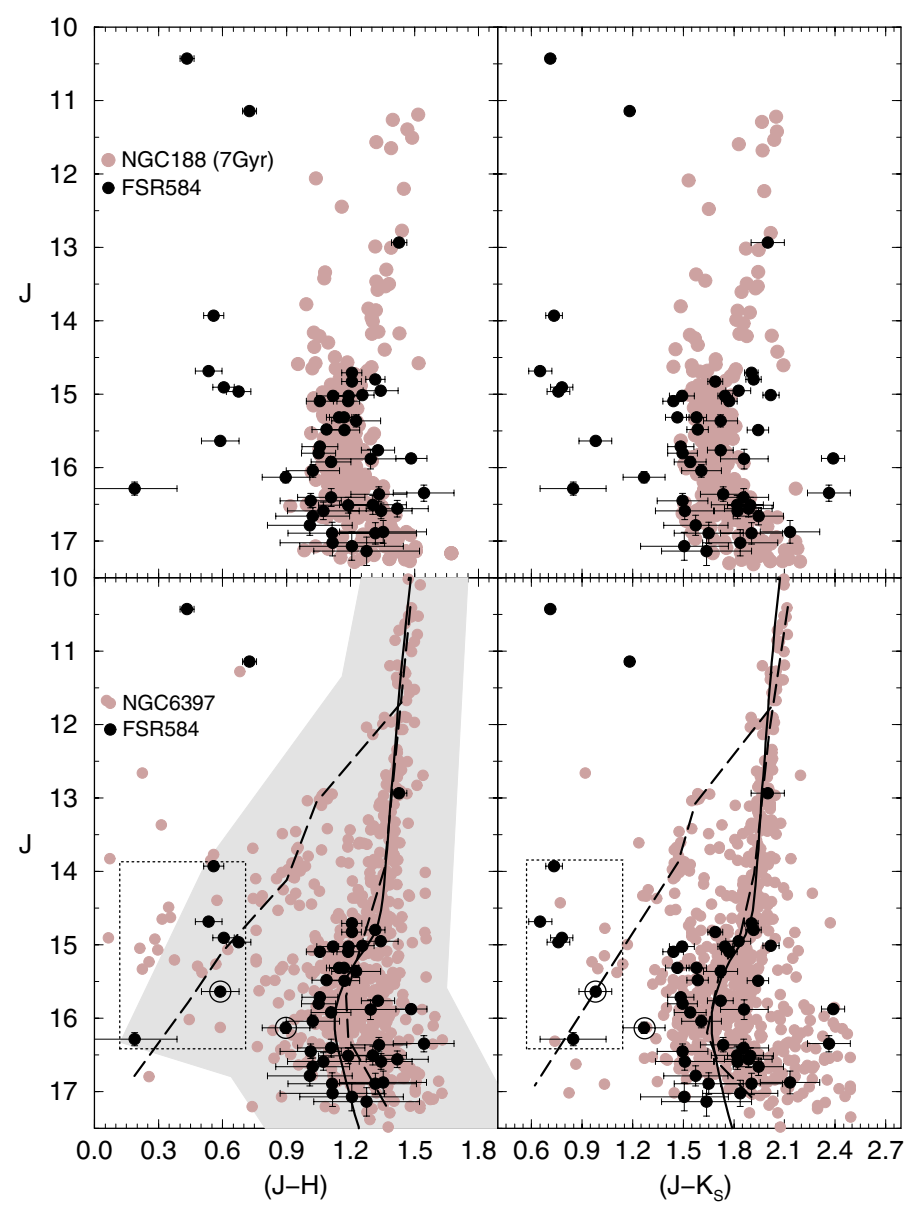

Fig. 3. Top: $R<1^{\prime}$ CMD of FSR 584 (black circles) compared to a CMD of the 7 Gyr open cluster NGC 188 (gray). Bottom: comparison with the $R<3^{\prime}$ CMD of the GC NGC 6397 (gray) and corresponding mean locus (dashed line). Solid line: $10 \mathrm{Gyr}$ Padova isochrone with $[\mathrm{Fe} / \mathrm{H}]=-2.0$. Dotted rectangle: locus of probable HB stars in FSR 584. Shaded area: colour-magnitude filter used to build the stellar radial density profile. Field decontamination would remove the 2 encircled stars.

CMD morphology of the $R<1^{\prime}$ region of FSR 584 is similar to that of the GC NGC 6397 with metallicity $[\mathrm{Fe} / \mathrm{H}]=-1.95$ (Harris 1996), built with 2MASS photometry of the $R<3^{\prime}$ region. As a reference for the MS, TO and red giant branch (RGB) sequences, we use a 10 Gyr Padova (Girardi et al. 2002) isochrone of $[\mathrm{Fe} / \mathrm{H}]=-2.0$. Young clusters, as a rule, have less densely populated TOs (Bonatto et al. 2006) and do not account for the blue HB stars. The results are consistent with a metalpoor GC, containing some blue HB stars. FSR 584 appears to be similar to the low-mass GCs AL 3, Palomar 13 (Siegel et al. 2001), and AM 4 (Inman \& Carney 1987), which contain about 10 giants.

Isochrone fits are independently applied to CMDs in both colours to derive photometric parameters (Fig. 3). The fits imply a reddening $E(J-H)=0.93 \pm 0.05$, which converts to $E(B-V)=3.0 \pm 0.2$, and $A_{V}=9.2 \pm 0.6^{3}$. The distance of FSR 584 from the Sun is $d_{\odot}=1.4 \pm 0.2 \mathrm{kpc}$, virtually crossing the plane at a height of $\approx 20 \mathrm{pc}$. Adopting $R_{\odot}=7.2 \mathrm{kpc}$ as the Sun's distance to the Galactic center (Bica et al. 2006b), we find that FSR 584 is located $\approx 1 \mathrm{kpc}$ beyond the Solar circle. Among

\footnotetext{
${ }^{3}$ Reddening transformations are $A_{J} / A_{V}=0.276, A_{H} / A_{V}=0.176$, $A_{K_{\mathrm{S}}} / A_{V}=0.118$, and $A_{J}=2.76 \times E(J-H)$ (Dutra et al. 2002), with $R_{V}=3.1$.
} 

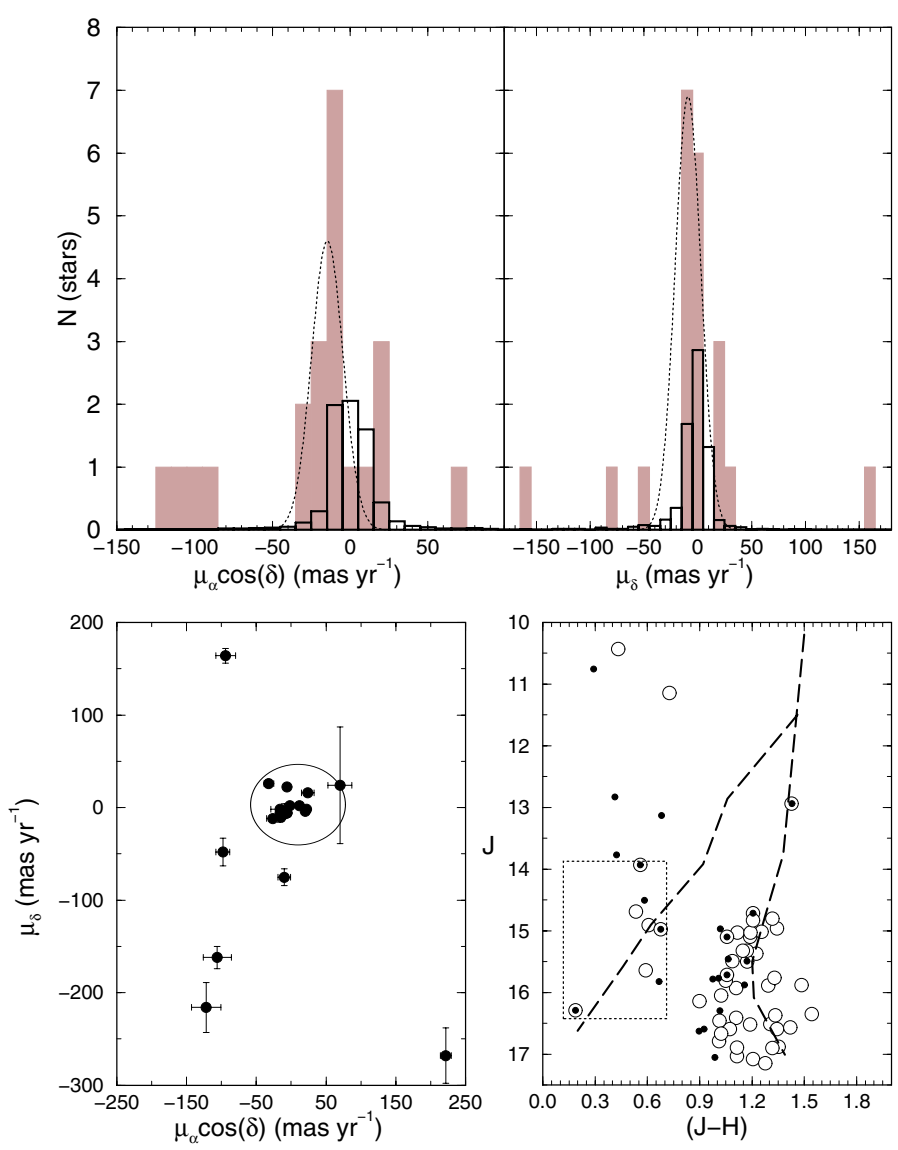

Fig. 4. Top: proper motions of the stars within $R<2^{\prime}$ (shaded histogram) compared to those at $40^{\prime}<R<50^{\prime}$ (empty), corrected for the different solid angles. Dotted line: Gaussian fit to the PM distribution of the central region. Bottom-left: PM components of the $R<2^{\prime}$ stars of FSR 584. Those located in the clump (circle) occur mostly in the MS and HB of FSR 584 (circles with dot - right panel). Dashed line: fiducial line of NGC 6397 (from Fig. 3).

the optical GCs, M4 and NGC 6397 are located at $d_{\odot}=2.2$ and $d_{\odot}=2.3 \mathrm{kpc}$, respectively (Harris 1996). The IR GC GLIMPSEC01, is also quite close at $d_{\odot} \approx 4 \mathrm{kpc}$ (Kobulnicky et al. 2005). This implies that FSR 584 is the nearest GC.

We further tested the nature of FSR 584 with proper motion data from NOMAD ${ }^{4}$. Since the correspondence between 2MASS and NOMAD is not complete, we extracted the PM of the stars within $R<2^{\prime}$ of FSR 584 to increase statistics. Besides, the stellar density profile of FSR 584 for $R<2^{\prime}$ presents a high contrast with respect to the background (Fig. 5). The PM distributions of these stars present differences both in the number of stars per PM bin and average values, especially in right ascension, with respect to the distant comparison ring $40^{\prime}<R<50^{\prime}$ (Fig. 4). The average values for the central region are $\left\langle\mu_{\delta}\right\rangle=$ $-9.1 \pm 1.4 \mathrm{mas} \mathrm{yr}^{-1}$ and $\left\langle\mu_{\alpha} \cos (\delta)\right\rangle=-14.7 \pm 3.3 \mathrm{mas} \mathrm{yr}^{-1}$. Most of the $R<2^{\prime}$ stars populate a clump in the PM plane, $\left|\mu_{\delta}\right|=\left|\mu_{\alpha} \cos (\delta)\right| \lesssim 35 \mathrm{mas} \mathrm{yr}^{-1}$, as expected for a star cluster. In the $R<1^{\prime}$ observed CMD of FSR 584, these stars occur mostly in the MS/TO and HB sequences (Fig. 4). Although in smaller number because PM data are from the optical, red stars essentially share the same PM as the blue stars. This motion in common supports the scenario where the bluer stars (HB)

\footnotetext{
4 http://vizier.u-strasbg.fr/viz-bin/ VizieR?-source=I/297
}

belong to the cluster, which is compatible with FSR 584 being a metal-poor halo GC. Assuming the average PM values of these stars as representative of FSR 584, we estimate that it is moving towards the Galactic plane at an angle of $\approx 71 \pm 5^{\circ}$, consistent with the motion of a halo object. In the case of an old open cluster, it would have very peculiar kinematics.

\subsection{Structural parameters of FSR 584}

The structure is investigated using the stellar radial density profile (RDP), built with colour-magnitude filtered photometry for $R<30^{\prime}$ (Bonatto \& Bica 2007). The filter (Fig. 3) removes contamination of stars with colours deviant from cluster sequences in the CMD. The resulting profile (Fig. 5) presents a prominent excess over the background especially for $R<4 \mathrm{pc}$. Most of the RDP follows King's (1966) law. However, the innermost point has a $1 \sigma$ excess density over the King fit, which might suggest post-core collapse. GCs with that structure occur mostly in the bulge, but some halo GCs like NGC 6397 also have it (Chernoff \& Djorgovski 1989; Trager et al. 1995).

Despite the central excess, the King fit provides a core radius of $R_{\text {core }}=0.3 \pm 0.1 \mathrm{pc}\left(\approx 0.75^{\prime}\right)$. The cluster limiting radius (the distance from the cluster center where RDP and background statistically merge) is $R_{\text {lim }}=4.5 \pm 0.5 \mathrm{pc}\left(\approx 11^{\prime}\right)$. Within uncertainties, the present value of $R_{\text {core }}$ is compatible with that in Froebrich et al. $(2007 \mathrm{a}), R_{\text {core }}=1^{\prime}$. They estimate a tidal radius of $R_{\text {tidal }}=50.6^{\prime}$, about 5 times larger than $R_{\text {lim }}$. However, dust, especially in the W 3 molecular cloud (Sect. 3 ), introduces dips in radial star counts for $R>30^{\prime}$ that precludes derivation of $R_{\text {tidal }}$ from the King fit. The inset of Fig. 5 displays the RDP of FSR 584 in the usual, background-subtracted presentation for GCs.

\section{Discussion}

An unusual property of FSR 584 is that it is projected in the direction of the large (diameter $\approx 50^{\prime}$ ), nearby molecular cloud/star-forming region W 3 (Carpenter et al. 2000). Within uncertainties, the distance of FSR 584 is compatible with $d_{\odot} \approx$ $1.88 \mathrm{kpc}$ (Kharchenko et al. 2005) for the cluster IC 1805 in the neighboring cloud W 5, at a comparable distance (Carpenter et al. 2000).

Angular positions and sizes of the known embedded clusters in W 3 are compared with those of FSR 584 in Fig. 6. The embedded clusters are from Carpenter et al. (2000), Megeath et al. (1996), Bica et al. (2003a,b), and Tieftrunk et al. (1998). FSR 584 is spatially separated from the known embedded clusters in W 3.

An optical nebula is present in blue and red XDSS and DSS images (Fig. 1). This reflection and/or ionizing nebula might be associated with the cluster. The latter peculiar relation with an old cluster is possible since blue HB stars normally attain $10^{4} \mathrm{~K}$, and hot ones may reach $2 \times 10^{4} \mathrm{~K}$ (Moeler et al. 1977). Most of the nebula is contained within $R \approx 2.5^{\prime}(\approx 1 \mathrm{pc})$. Following photo-dissociation, $10^{4} \mathrm{~K}$ stars can ionize gas in a comparable volume for densities of $\sim 1 \mathrm{H}$ atom $\mathrm{cm}^{-3}$, while $2 \times 10^{4} \mathrm{~K}$ stars may ionize gas as far as $8-10 \mathrm{pc}$.

FSR 584 appears to be a halo GC moving towards the plane (Sect. 2.2). Besides disk-shocking, it might be suffering additional tidal stress due to its proximity to the massive W $3, \mathrm{~W} 4$ and W 5 molecular cloud complex (Wielen 1991). W 3 itself is relatively massive with $\approx 10^{5} M_{\odot}$ (Lada et al. 1987). In the long term, dynamical heating of a GC is expected as a consequence 


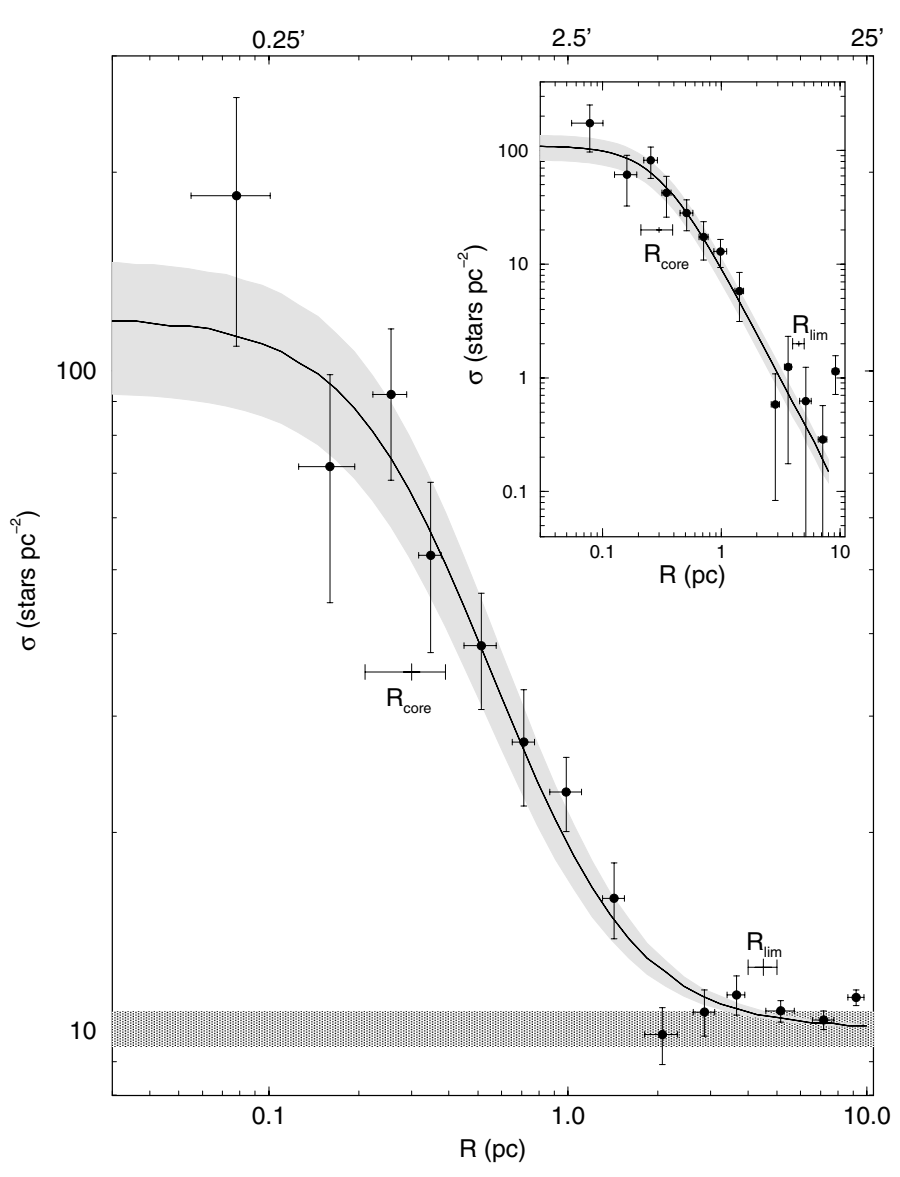

Fig. 5. Stellar RDP (filled circles) of FSR 584 in absolute scale. Solid lines: best-fit King profile. Horizontal shaded region: offset field stellar background level. Core and limiting radii are indicated. Gray regions: $1 \sigma$ King fit uncertainty. The angular scale is in the upper abscissa. Inset: background-subtracted RDP together with King fit.

of tidal interactions by shocks due to disk and bulge crossings, as well as encounters with massive molecular clouds. This enhances the rate of low-mass star evaporation and accelerates the process of core collapse (Djorgovski \& Meylan 1994). A combination of these effects could enhance the cluster dynamical evolution (Bonatto \& Bica 2007, and references therein) with core contraction and halo expansion (e.g. Goodwin \& Bastian 2006). This might explain a central density excess over the King profile (Fig. 5) and, at the same time, account for the presence of cluster stars driven to distances of up to $\sim 20^{\prime}(\sim 8 \mathrm{pc})$ from the cluster center (Fig. 2). A similar effect was detected in the intermediateage open cluster BH 63 (Bica et al. 2006a), which shows a postcore collapse structure, probably because of interaction with a neighboring dust cloud.

\section{Concluding remarks}

FSR 584 was found by Froebrich et al. (2007a) in an automated star cluster survey using 2MASS. The near-IR CMD morphology (Sect. 2.2) combined to the stellar radial density profile (Sect. 2.3) provide convincing evidence that FSR 584 is an old star cluster. Together with these results, PMs compatible with the halo suggest an as yet unidentified GC in the Galaxy. Deeper IR photometry complemented with spectroscopy are necessary to settle the nature of FSR 584, as a GC (old or young) or an old open cluster with peculiar kinematics. We point out that

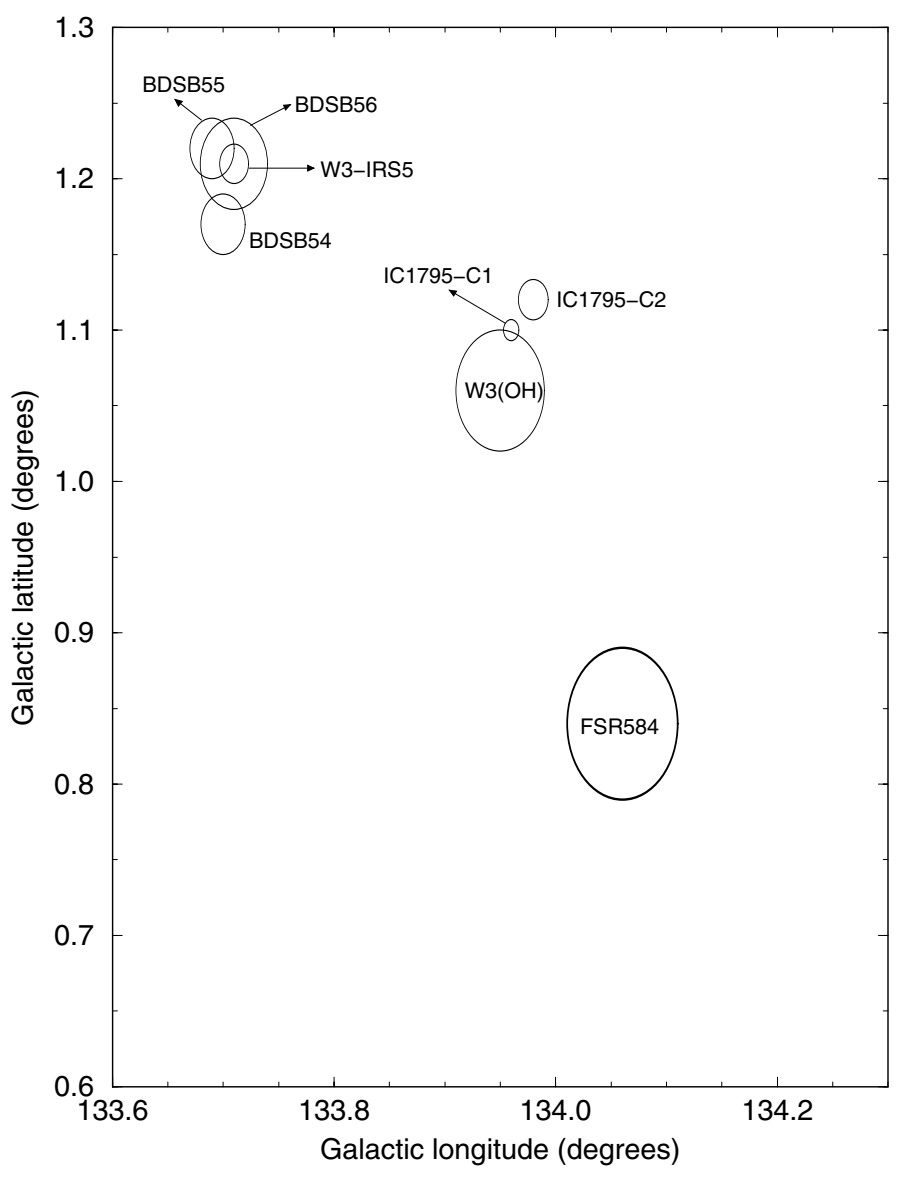

Fig. 6. Angular distribution and size of embedded clusters in the W 3 cloud together with those of FSR 584. The $3^{\prime}$ radius of FSR 584 in this figure corresponds basically to the region containing the bulk of cluster stars (Fig. 5), thus encompassing the cluster region shown in the 2MASS image (Fig. 1). Orientation as in Fig. 1.

young GCs such as Pal 1 (Rosenberg et al. 1998) and Whiting 1 (Carraro 2005) are low-mass objects with hardly any giants and essentially no HB stars. This is also the case for the recently discovered low-mass halo GCs Koposov 1 and 2 (Koposov et al. 2007), and the additional ones listed in Sect. 1.

The current census indicates the presence of 159 GCs so far detected in the Milky Way (Sect. 1). However, a fundamental question is how to define a Galactic GC. The census includes a few dwarf galaxy nuclei, e.g. $\omega$ Cen and M 54 (Bica et al. 2006b and references therein). The recently discovered SDSS stellar systems in the outer halo (Sect. 1) might be dwarf galaxies. Some clusters not far from the plane, like Palomar 1 with a relatively young age (Rosenberg et al. 1998), might be old open clusters.

In the case of a GC, FSR 584 would be the 160th detected in the Galaxy. FSR 584 is remarkably close to the Sun at $d_{\odot}=$ $1.4 \pm 0.2 \mathrm{kpc}(\approx 1 \mathrm{kpc}$ beyond the Solar circle) $)$, thus resulting as the nearest GC. It is located close to the Galactic plane $(Z \approx$ $20 \mathrm{pc}$ ), and is moving towards the plane, according to the PM results (Sect. 2.2).

The photometric and structural parameters of FSR 584 are consistent with a Palomar-like halo GC, i.e. a low mass GC containing some giants. The TO of FSR 584 is readily detected in the present near-IR CMDs (consistent with the proximity), as well as the MS and possibly some blue HB stars. A metallicity of $[\mathrm{Fe} / \mathrm{H}] \approx-2.0$ is estimated, together with an absorption of $A_{\mathrm{V}}=9.2 \pm 0.6$. Its Palomar-like nature, together with the 
fact that the bulk of its stars require IR photometry due to a relatively high absorption, can explain why it has so far been overlooked. FSR 584 is projected in the direction of the molecular cloud W 3 , and evidence is found that they actually interact, which appears to be the major source of absorption. Disk shocking and the possible encounter with a massive cloud may have enhanced the dynamical evolution of FSR 584, as suggested by a central density excess in the radial density profile. Regardless of the globular or open cluster nature, the old age of FSR 584 makes it a promising dynamical laboratory to search for significant stellar losses and/or disruption related to the potentially damaging environment.

The fact that in less than one year, five new Palomar-like GCs have been identified, AL 3 in the bulge, SEGUE 1, Koposov 1 and 2, and now possibly FSR 584 in the halo, suggests that the number of low-mass GCs may be considerably larger than previously thought.

Acknowledgements. We thank the anonymous referee for suggestions. We acknowledge partial support from $\mathrm{CNPq}$ and FAPESP (Brazil), and MURST (Italy).

\section{References}

Belokurov, V., Evans, N. W., Irwin, M. J., et al. 2007, ApJ, 658, 337 Bica, E., Dutra, C. M., \& Barbuy, B. 2003a A\&A, 397, 177 Bica, E., Dutra, C. M., Soares, J., \& Barbuy, B. 2003b A\&A, 404, 223 Bica, E., Bonatto, C., \& Blumberg, R. 2006a, A\&A, 460, 83 Bica, E., Bonatto, C., Barbuy, B., \& Ortolani, S. 2006b, A\&A, 450, 105 Bonatto, C., \& Bica, E. 2007, MNRAS, 377, 1301

Bonatto, C., Bica, E., \& Girardi, L. 2004, A\&A, 415, 571

Bonatto, C., Bica, E., \& Santos Jr., J. F. C. 2005, A\&A, 433, 917

Bonatto, C., Bica, E., Ortolani, S., \& Barbuy, B. 2006, A\&A, 453, 121
Carpenter, J. M., Heyer, M. H. \& Snell, R. L. 2000, ApJS, 130, 381 Carraro, G. 2005, ApJ, 621, L61

Chernoff, D., \& Djorgovski, S. 1989, ApJ, 339, 904

Djorgovski, S., \& Meylan, G. 1994, AJ, 108, 1292

Dutra, C. M., Santiago, B. X., \& Bica, E. 2002, A\&A, 383, 219

Froebrich, D., Scholz, A. \& Raftery, C. L. 2007a, MNRAS, 374, 399

Froebrich, D., Meusinger, H., \& Scholz, A. 2007b, MNRAS, 377, L54

Girardi, L., Bertelli, G., Bressan, A., et al. 2002, A\&A, 391, 195

Gnedin, O. Y., \& Otriker, J. P. 1997, ApJ, 474, 223

Goodwin, S. P., \& Bastian, N. 2006, MNRAS, 373, 752

Harris, W. E. 1996, AJ, 112, 1487

Inman, R. T., \& Carney, B. W. 1987, AJ, 83, 1166

Khalisi, E., Amaro-Seoane, P., \& Spurzem, R. 2007, MNRAS, 374, 703

Kharchenko, N. V., Piskunov, A. E., Röser, S., Schilbach, E., \& Scholz, R.-D. 2005, A\&A, 438, 1163

King, I. 1966, AJ, 71, 64

Kobulnicky, H. A., Monson, A. J., Bickalew, B. A., et al. 2005, AJ, 129, 239

Koposov, S., de Jong, J. T. A., Belokurov, V., et al. 2007, ApJ, submitted [arXiv:0706.0019]

Lada, C. J., Elmegreen, B. G., Cong., H.-I., \& Thaddeus, P. 1987, ApJ, 226, L39

Lamers, H. J. G. L. M., Gieles, M., Bastian, N., et al. 2005, A\&A, 441, 117

Landsman, W., Bohlin, R. C., Neff, S. G., et al. 1998, AJ, 116, 789

Mackey, A. D., \& van den Bergh, S., 2005, MNRAS, 360, 631

Megeath, S. T., Herter, T., Beivhman, C., et al. 1996, A\&A, 307, 775

Moeler, S., Heber, U., \& de Boer, K. S. 1977, AA, 294, 65

Ortolani, S., Bica, E., \& Barbuy, B. 2006, ApJ, 646, L115

Ortolani, S., Rosino, L., \& Sandage, A. 1985, AJ, 90, 473

Rosenberg, A., Saviane, I. Piotto, G., Aparicio, A., \& Zaggia, S. R. 1998, AJ, 115,648

Sakamoto, T., \& Hasegawa, T. 2006, ApJ, 653, L29

Siegel, M. H., Majewski, S. R., Cudworth, K. M., \& Takamiya, M. 2001, AJ, 121, 935

Tieftrunk, A. R., Megeath, S. T., Wilson, T. L., \& Rayner, J. T. 1998, A\&A, 336, 991

Trager, S., King, I. R., \& Djorgovski, S. 1995, AJ, 109, 218

van den Bergh, S. 1991, ApJ, 375, 594

Wielen, R. 1991, in The Formation and Evolution of Star Clusters, ed. K. Janes, ASP Conf. Ser. 13, San Francisco, CA, 342

Willman, B., Blanton, M. R., West, A. A., et al. 2005, AJ, 129, 2692 